\title{
A Peculiar Case of a Dog Bite
}

\author{
Vilashini Arul Devah, Ana Pokrajac, Mark Savage, Isha Malik
}

Department of Endocrinology, North Manchester General Hospital, Manchester, M8 5RB UK.

\section{INTRODUCTION}

We present a case of ectopic-ACTH syndrome, a rare cause of Cushing's syndrome, where initial presentation of dog bite proved to be misleading and when a diagnosis of Cushing's syndrome was made, despite extensive diagnostic procedures, finding the source of ACTH secretion, was challenging.

\section{PRESENTATION}

59-year old male, ex-lorry driver and a current smoker presented to Emergency Assessment Unit (EAU) in April 2012 :

- Reported history of generalised facial and ankle oedema since a dog bite 6 weeks back, for which had received penicillin.

- Treated as angioedema (Prednisolone and antihistamine) and discharged.

\section{PMH:}

- November 2011- Investigations (OGD \& Colonoscopy) organised to investigate abdominal pain \& altered bowel but patient failed to attend appointments.

-2005- Lung lesion on CT Thorax - Benign lung polyp on histology

Short EAU assessments ( 5-between 08/05/2012 \&

$11 / 06 / 12$ ) with progressive symptoms

-Worsening anasarca

- Breathlessness on exertion

- Noted to have mild hypertension, hypokalaemia, few high random blood glucose levels

-Differential diagnosis of Nephrotic syndrome; Congestive Heart Failure; Conn's syndrome and Vasculitis considered and ruled out by doing relevant investigations

-Also, recorded on couple of occasions to have " Cushingoid facies"

- Furosemide, Spironolactone initiated and referred to Endocrine outpatient clinic for further investigations.

\section{Readmitted-15/06/2012 with}

- Definite cushingoid appearance, new established diagnosis of diabetes \& hypertension and persistent hypokalemia

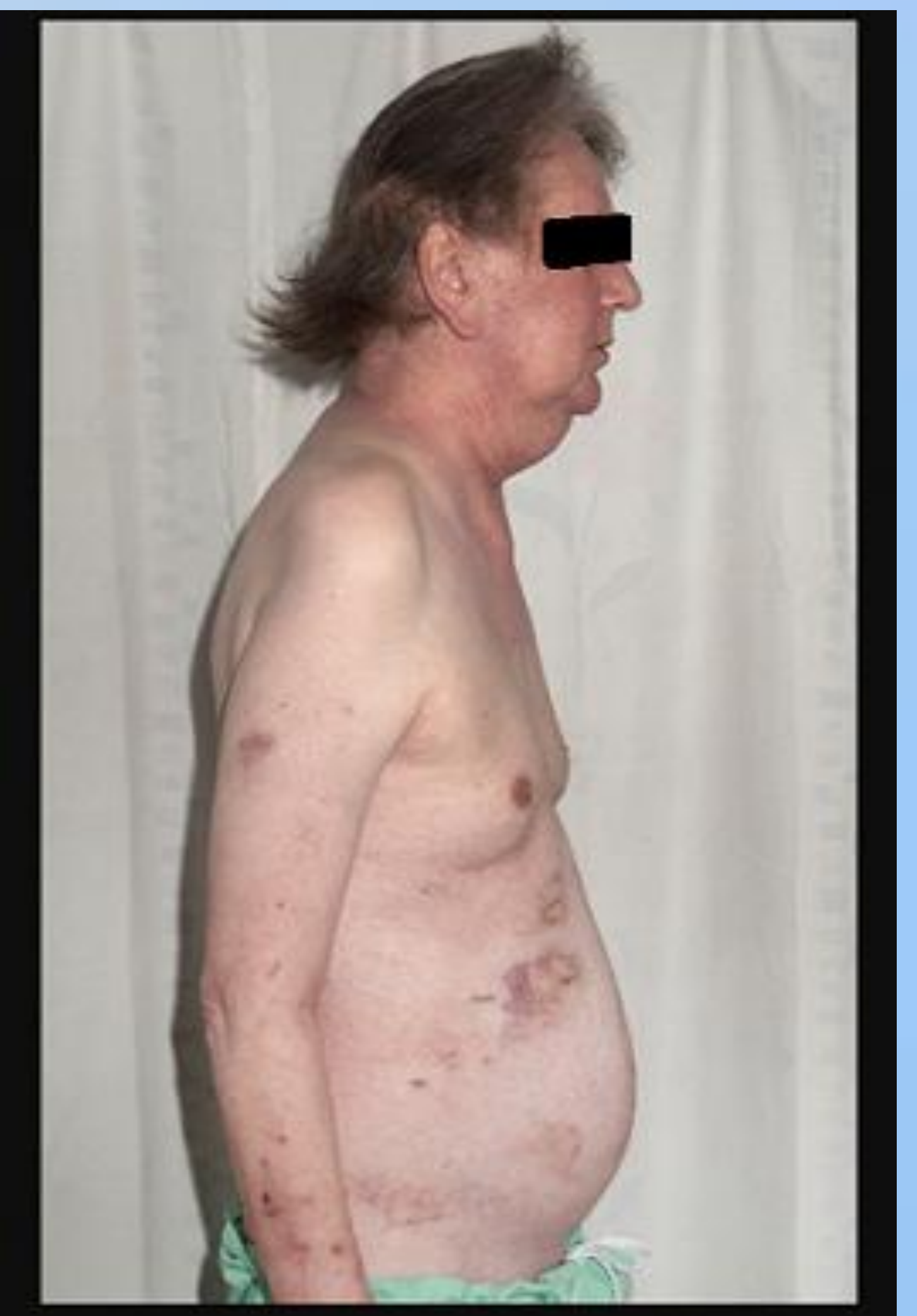

- Started on Metformin \& Insulin

- Urgent inpatient investigations for Cushing's syndrome organised.

\section{INITIAL ENDOCRINE INVESTIGATIONS}

- Low dose dexamethasone suppression test

\begin{tabular}{l|l|l} 
& Baseline & $\mathbf{4 8}$ hrs Post-Dex \\
\hline Cortisol (n mol/l) & 2203 & 2318 \\
\hline ACTH $(\mathrm{ng} / \mathrm{l})$ & $<5$ & \\
\hline
\end{tabular}

Given short history of progressive symptoms-

Ectopic ACTH-syndrome suspected

- ACTH-precursors (POMC): 610 p mol/l $(\mathrm{N}<100)$ - ACTH (different lab)=13 ng $/ 1(<50)$

\section{OTHER INVESTIGATIONS}

- TFT and Prolactin - normal

- IGF-1 (<25 mcg/l)

- LH; FSH -low

- MRI pituitary - normal

- Calcium- normal

- High PTH (91ng/l)

- Low Vitamin D levels (6.9 ug /l)

- Urinary 5-HIAA- normal

- Plasma metanephrine - normal

\section{INVESTIGATIONS- IMAGING \& HISTOLOGY}

CT scan and MRI abdomen

- Metastatic liver lesions; bilateral adrenal gland enlargement/ multiple nodules; no pancreatic lesions
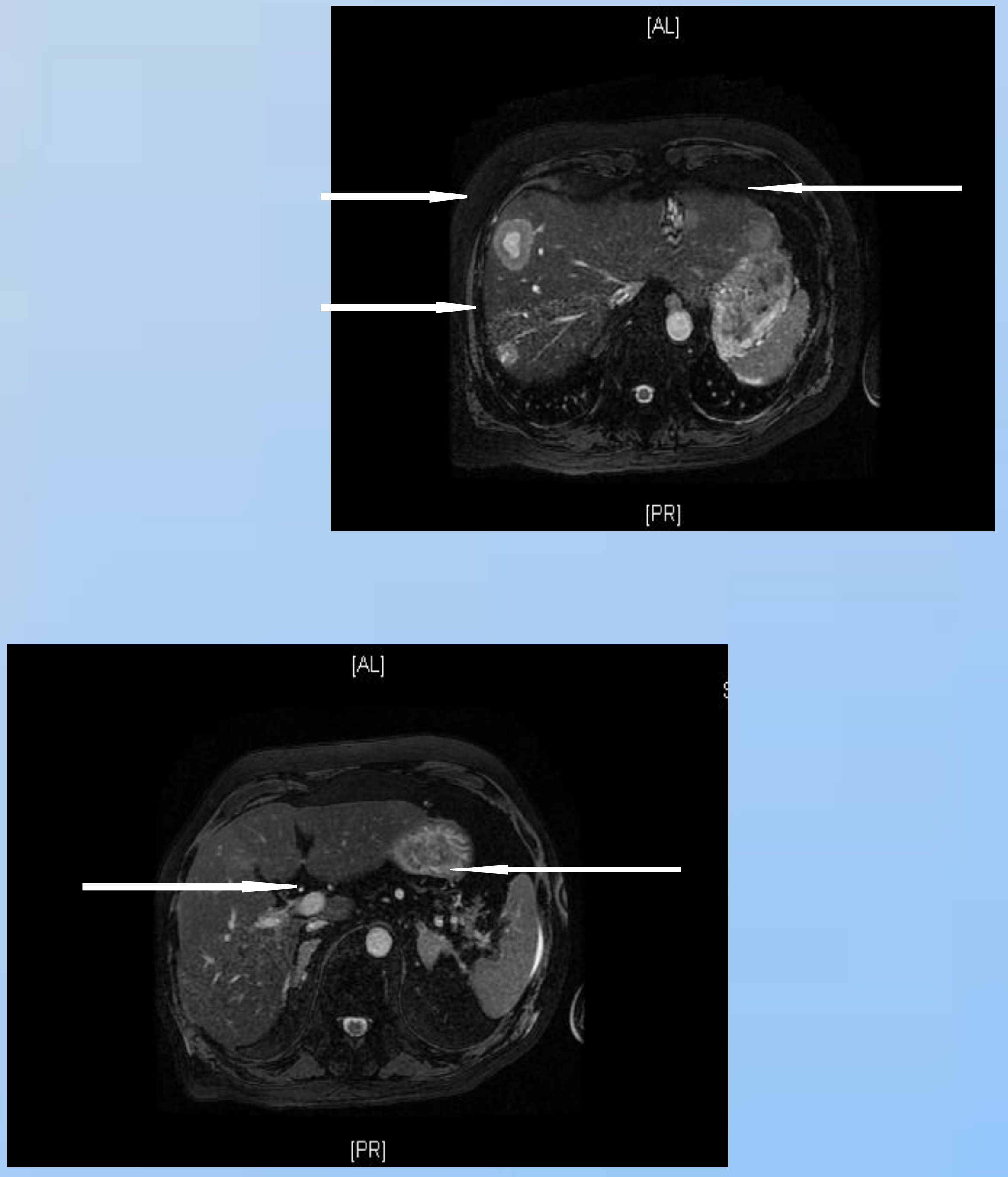

\section{SEARCH FOR THE PRIMARY}

Liver Biopsy:

Metastatic neuroendocrine tumour (Ki67 25\%); Grade 3 CDX2 positive - suggested primary sites:

$$
\begin{aligned}
& \text { 1. Stomach } \\
& \text { 2. Small intestine } \\
& \text { 3. Possibly pancreas }
\end{aligned}
$$

\section{NM Octreotide scan:}

Findings not typical for somatostatin receptor +ve disease

Upper GI endoscopy:

Candidiasis; No lesion

\section{Fasting gut hormones - elevated}

$\mathrm{CART}=469 \mathrm{p} \mathrm{mol} / \mathrm{L}(<85)$

Chromogranin $\mathrm{A}=247 \mathrm{p} \mathrm{mol} / \mathrm{L}(<60)$

Chromogranin $\mathrm{B}=216 \mathrm{p} \mathrm{mol} / \mathrm{L}(<150)$

\section{TREATMENT}

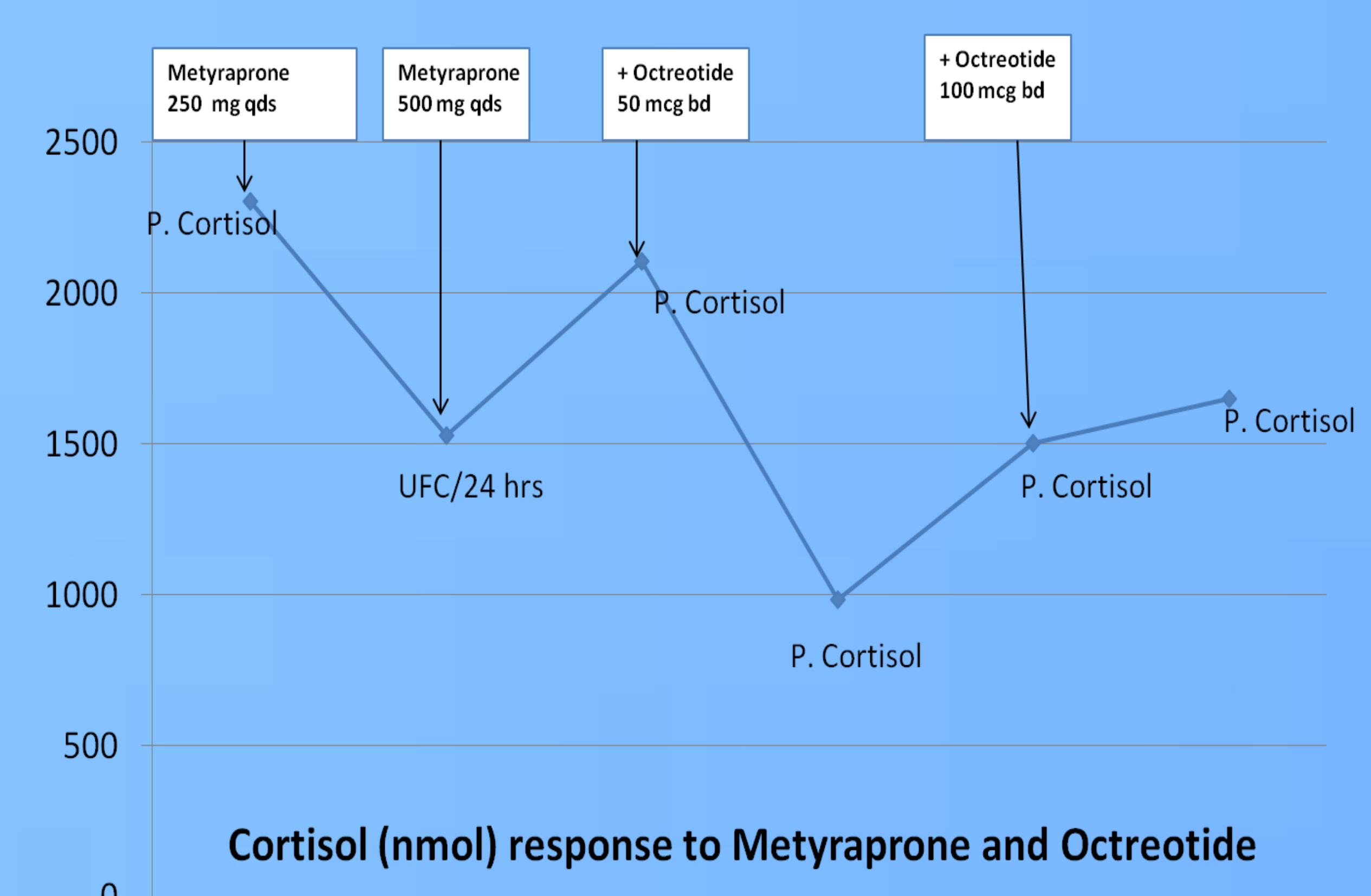

\section{SEARCH FOR THE PRIMARY}

PET Scan:

High metabolic activity within the tail of pancreas, bilateral adrenal lesions and liver suspicious of malignancy

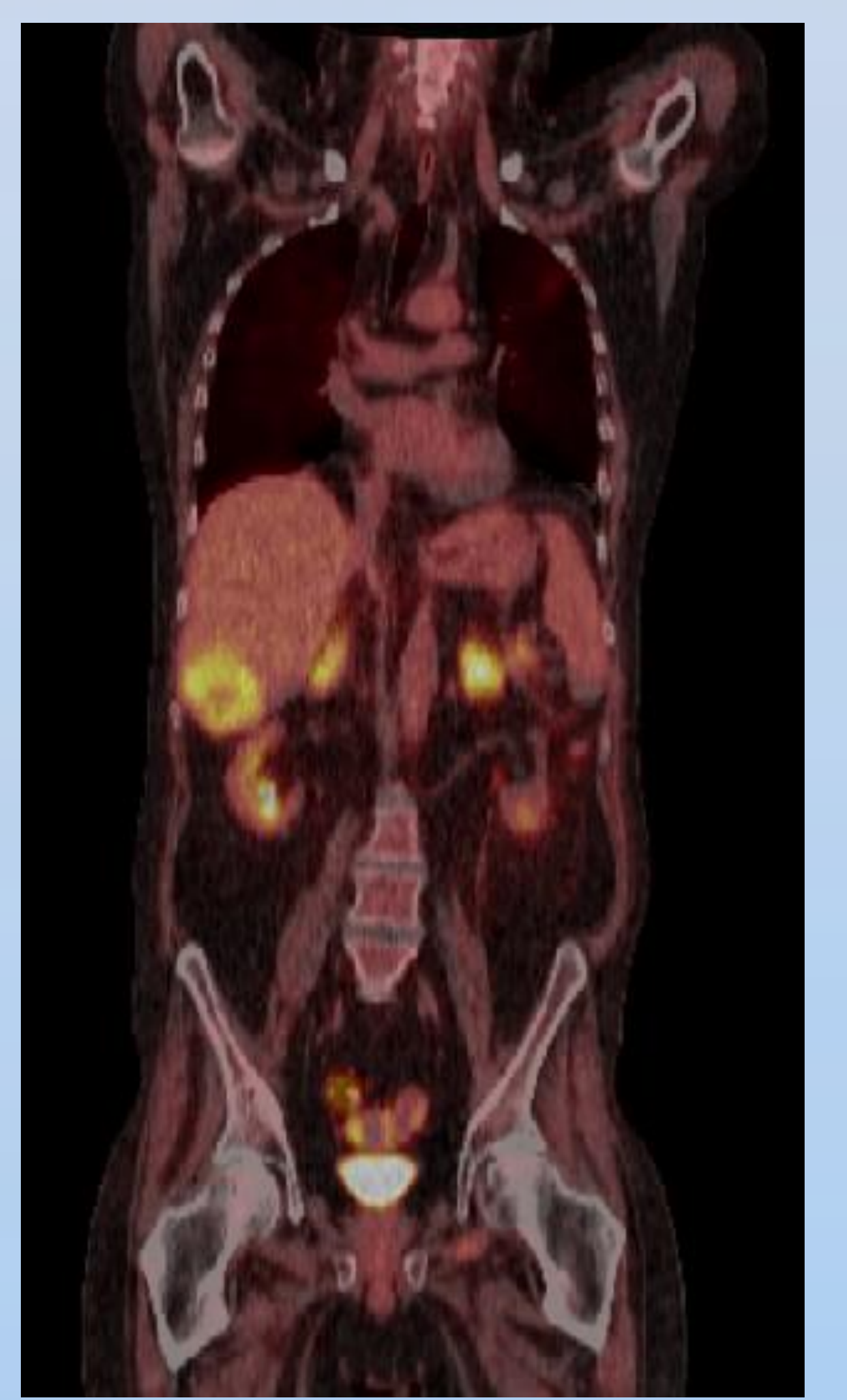

\section{SURGERY}

- Bilateral adrenalectomy; splenectomy; tail of pancreas resection- 07/08/2012 (based on Neuroendocrine MD' discussion \& recommendation)

- Histology: Grade 3, poorly differentiated endocrine carcinoma pT3 pN1 pM1, (Ki67 40-50\%)

\section{DIAGNOSIS}

Ectopic ACTH syndrome caused by Pancreatic Neuroendocrine tumour (NET) secreting ACTH precursors

\section{POST-OP COURSE}

- On Hydrocortisone and Fludrocortisone

- Received appropriate vaccinations; long-term antibiotics prophylaxis

- Off metformin, insulin, anti-hypertensive agents, diuretics

- Repeat CT scan showed increasing size of liver metastatic lesions- transarterial chemoembolisation being considered.

\section{DISCUSSION}

- Ectopic secretion of ACTH is rare contributing to $10 \%-15 \%$ cases of endogenous Crushing's syndrome ${ }^{1}$.

- Ectopic ACTH secretion has been most commonly reported from small cell lung carcinoma and less commonly from indolent tumours such as bronchial, thymic, pancreatic carcinoids and thyroid medullary carcinoma ${ }^{2}$.

- Ectopic ACTH production by the pancreatic NET is relatively rare, and patients with this tumour show poor prognosis ${ }^{3}$.

- Tumours causing the ectopic ACTH syndrome tend to secrete a disproportionately greater proportion of POMC precursors, and these may be their major product $^{4}$

- Inappropriate repression or expression on certain genes, causes these tumours to secrete ACTH and other POMC-derived peptides ${ }^{4}$.

- ACTH secretion from malignant ectopic sources is not inhibited by dexamethasone. In rare patients, glucocorticoids increase tumour secretion of $\mathrm{ACTH}$. This is important to remember when interpreting dexamethasone suppression tests ${ }^{5}$.

\section{CONCLUSION}

Short progressive history, association of new diagnosis of hypertension and glucose intolerance, unexplained hypokalemia should raise the index of suspicion for ectopic ACTH-precursor syndrome.

When an ectopic ACTH-dependent Cushing's syndrome is suspected, but ACTH is not in the diagnostic range, and infact as was in this case, is actually suppressed, it is important to ensure that the assay used to measure ACTH has a high degree of crossreactivity of the ACTH-precursors in the ACTH assay or that a separate specific assay for ACTH precursors is used. 